\title{
Organization of Vomeronasal Sensory Coding Revealed by Fast Volumetric Calcium Imaging
}

\author{
Diwakar Turaga and Timothy E. Holy \\ Department of Anatomy and Neurobiology, Washington University in St. Louis School of Medicine, St. Louis, Missouri 63110
}

\begin{abstract}
A long-standing goal in neuroscience is to perform exhaustive recording of each neuron in a functional local circuit. To achieve this goal, one promising approach is optical imaging of fluorescent calcium indicators, but typically the tens or hundreds of cells imaged simultaneously comprise only a tiny percentage of the neurons in an intact circuit. Here, we show that a recent innovation, objective-coupled planar illumination (OCPI) microscopy, permits simultaneous recording from three-dimensional volumes containing many thousand neurons. We used OCPI microscopy to record chemosensory responses in the mouse vomeronasal epithelium, for which expression of hundreds of receptor types implies high functional diversity. The implications of this diversity for sensory coding were examined using several classes of previously reported vomeronasal ligands, including sulfated steroids. A collection of just 12 sulfated steroids activated more than a quarter of the neurons in the apical vomeronasal epithelium; unexpectedly, responses were functionally organized into a modest number of classes with characteristic spatial distribution. Recording from a whole sensory system thus revealed new organizational principles.
\end{abstract}

\section{Introduction}

Neurophysiology is frequently compared with the parable of the blind men and an elephant, in which the exploration of small parts of the elephant leads to very disparate views of its nature. This state of affairs is a consequence of technical barriers that limit the ability to record from more than a few tens or hundreds of neurons simultaneously, typically a small portion of the activity required to represent a naturalistic stimulus. For sensory systems like olfaction-in which odorant information is encoded via the ligand-binding properties of families of hundreds or thousands of distinct receptor types (Buck and Axel, 1991)the limited scale of current recordings poses a major challenge. In principle, natural behaviors may be triggered by a very small percentage of cells, or might alternatively depend upon the collective activity of the full population. With only a partial view of the sensory information possessed by the animal, connecting molecular cues to circuits and behavior remains, particularly for mammals, a major challenge.

One of the most promising approaches to circumvent these barriers is calcium imaging. However, for intact tissue the most commonly used imaging techniques, two-photon and confocal microscopy, suffer from slow throughput due to the requirement for point-scanning. Consequently, there has been considerable

\footnotetext{
Received Oct. 23, 2011; revised Nov. 21, 2011; accepted Dec. 2, 2011.

Author contributions: D.T. and T.E.H. designed research; D.T. performed research; D.T. and T.E.H. analyzed data; D.T. and T.E.H. wrote the paper.

This study was funded by NIH-NINDS/NIAAA Grant R01 NS068409, NIH-NIDCD Grant R01 DC005964, and NIH Director's Pioneer Award DP1 0 D006437 (T.E.H.). We thank Ron Yu for the GCaMP2 mice; Julian Meeks for reagents and analyses; Hannah Arnson, Andreas Burkhalter, Vitaly Klyachko, Julian Meeks, Paul Taghert, Illya Tolokh, and Leslie Vosshall for comments on the manuscript; and the Holy lab for discussions.

T.E.H. has filed a patent application on OCPI microscopy.

Correspondence should be addressed to Timothy E. Holy, Campus Box 8108, 660 S. Euclid, St. Louis M0 63110. E-mail: holy@wustl.edu.

DOI:10.1523/JNEUROSCI.5339-11.2012

Copyright $\odot 2012$ the authors $\quad 0270-6474 / 12 / 321612-10 \$ 15.00 / 0$
}

interest in developing alternative fast-scanning microscopes (Wilt et al., 2009), but these do not produce full volumetric data without encountering the fundamental limit on the time spent dwelling over each voxel (Pawley, 2006). Recently we introduced a alternative approach, objective-coupled planar illumination (OCPI) microscopy (Holekamp et al., 2008). Because OCPI microscopy illuminates an entire focal plane at once, it allows parallel acquisition from a million (or more) voxels at once. Consequently, it offers optical sectioning at speeds far higher than point-scanning approaches.

Here we show that OCPI microscopy permits recording from thousands of neurons simultaneously in three-dimensional volumes, and that by successively recording from separate volumes, $>10,000$ neurons may be sampled in a single day. We apply this technique to study sensory coding in the vomeronasal epithelium, a sensory organ detecting chemical cues (sometimes called pheromones) relevant for social communication. Vomeronasal sensory neurons (VSNs) express $\sim 300$ distinct receptor types (Touhara and Vosshall, 2009), of which a single neuron abundantly expresses one or a few types. By sampling from thousands of neurons in a single imaging volume, OCPI microscopy permits nearly exhaustive recording of the sensory representations at the first stage of this sensory system.

We found that between a quarter and half of all apical VSNs, and a subset of basal VSNs, were activated by just 12 members of a recently reported family of ligands, sulfated steroids (Hsu et al., 2008; Nodari et al., 2008). Given the size of the receptor families, one might expect such abundant responses to be extremely diverse, reflecting the action of many receptor types; surprisingly, the large majority of responses were organized into a small number of categories, whose functional characteristics correlated with their spatial position within the epithelium. We discuss the implications of these findings for vomeronasal sensation and as a model for comprehensive recording in the wider nervous system. 

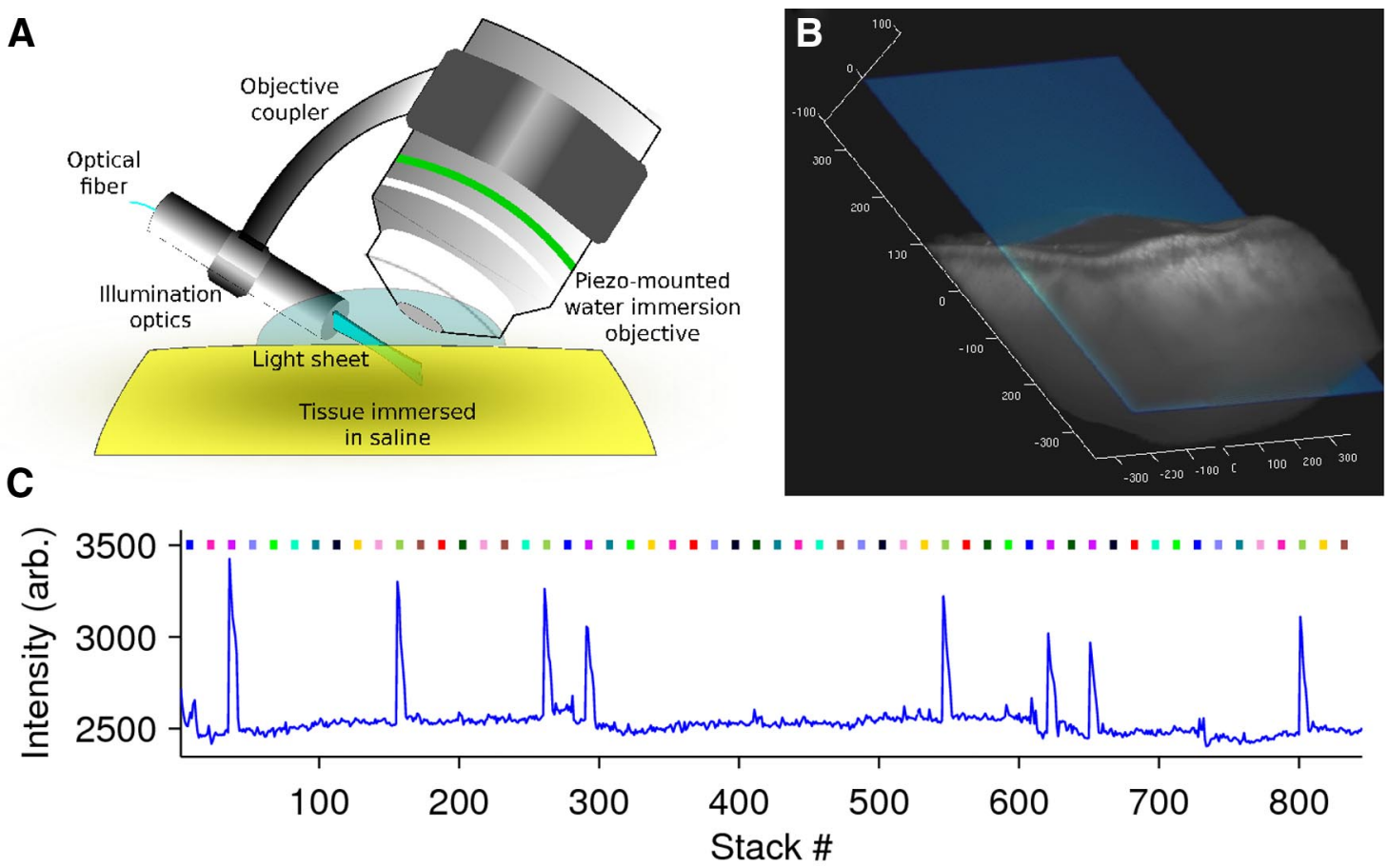

Pre (stack 155) Post (stack 156)
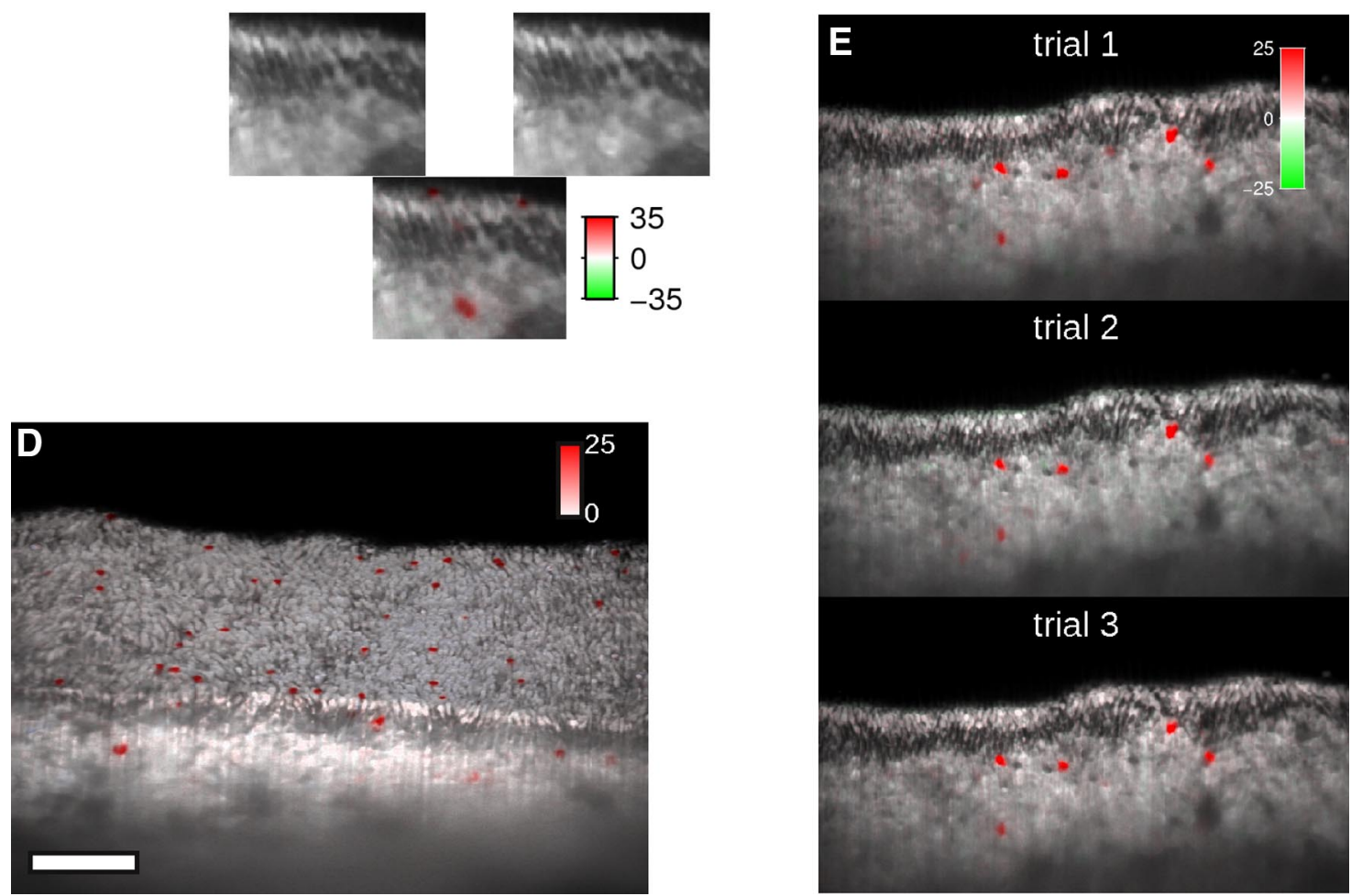

Figure 1. Sensory responses of VSNs visualized by OCPI microscopy. $A$, Schematic of OCPI microscope. The focal plane is illuminated by a thin sheet of light; to scan in three dimensions, the objective and illumination are moved by the piezoelectric manipulator. $\boldsymbol{B}$, Volume of the VNO scanned in $2 \mathrm{~s}$ (all units in micrometers). A single plane of illumination (blue) is shown. $\boldsymbol{C}$, Spatial and temporal representations of fluorescence changes. Below, raw fluorescence images in single optical sections from two successive stacks (dendritic knobs at surface, cell bodies with visible nuclei below); percentage fluorescence change is also represented in the bottom middle image using a red/green color scale. Above, mean fluorescence intensity as a function of time for the responsive cell body in the images below; delivery of different stimuli indicated by color bars (10 $\mu \mathrm{m}$ Q1570, olive; $10 \mu \mathrm{m}$ Q3910, dark magenta). Each stack is acquired in $2 \mathrm{~s}$, with $3 \mathrm{~s}$ to save the data. $\boldsymbol{D}$, Volume rendering of VNO under stimulation by Q1570. Red color scale indicates intensity of fluorescence change, which can be seen in isolated dendritic knobs at the tissue surface as well as in cell bodies deeper in the tissue. Scale bar, $100 \mu \mathrm{m}$. $\boldsymbol{E}$, Reproducibility in a single optical section across interleaved repeated presentations of responses to $10 \mu \mathrm{m} Q 1570$.

\section{Materials and Methods}

Animals. The tetO-GCaMP2 and OMP-IRES-tTA (on a C57BL/6 background) mice were a gift from Ron Yu, Stowers Institute for Medical Research, Kansas City, MO (Yu et al., 2004; He et al., 2008). The F1 generation from the cross were genotyped and the mice containing both the tetO-GCaMP2 and OMP-IRES-tTA genes were used in the experiments. The mice were housed and handled in accord with practices approved by the Washington University Animal Studies Com- 


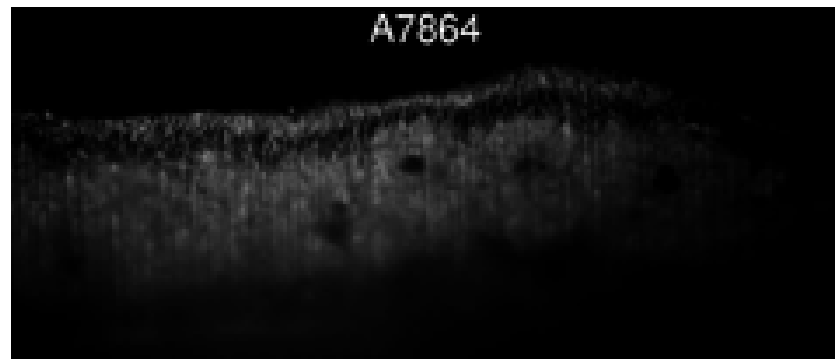

Movie 1. Fluorescence over time in a single optical section, while presenting chemical stimuli to the VNO. Stimulus presentation (valve opening) is coincident with the display of stimulus name. Both spontaneous and stimulated activity can be seen. A single trial of each stimulus is shown. Stimuli delivered to the apical surface of the tissue (top of tissue in movie, the location of the dendritic knobs). Movie constructed without enhancement; it consists of the raw images acquired by the camera. Simultaneously, 39 other optical sections were also being acquired. Movie rendered at 5 frames per second. Some artifacts of MPEG compression can be seen.

mittee. All the mice used in the experiments were female and 12-24 weeks of age.

Imaging. The imaging was performed on a custom Objective Coupled Planar Illumination microscope (Holekamp et al., 2008). Illumination was at $488 \mathrm{~nm}$, the light sheet was $\sim 5 \mu \mathrm{m}$ thick, and the objective was a water-immersion $0.5 \mathrm{NA} 20 \times$ (Olympus). The camera was an iXon DV885-KCS-VP cooled EM-CCD (Andor).

Solutions and stimuli. The excised vomeronasal organ (VNO) was continuously superfused with Ringer's solution (containing, in mM: $115 \mathrm{NaCl}, 5$ $\mathrm{KCl}, 2 \mathrm{CaCl}_{2}, 2 \mathrm{MgCl}_{2}, 25 \mathrm{NaHCO}_{3}, 10 \mathrm{HEPES}$, and 10 glucose). Highpotassium Ringer's (containing, in $\mathrm{mm}$ : $70 \mathrm{NaCl}, 50 \mathrm{KCl}, 2 \mathrm{CaCl}_{2}, 2 \mathrm{MgCl}_{2}$, $25 \mathrm{NaHCO}_{3}, 10 \mathrm{HEPES}$, and 10 glucose) was used to test the placement of the superfusion/stimulus delivery tube before the beginning of the experiment. Sulfated steroids were purchased from Steraloids and were stored at $-20^{\circ} \mathrm{C}$ in solid form. We used the following compounds, referenced by their Steraloids catalog number: A6940, 4-androsten-17 $\alpha$-ol-3-one sulfate (epitestosterone sulfate); A7010, 4-androsten-17 $\beta$-ol-3-one sulfate (testosterone sulfate); A7864, 5-androsten-3 $\beta, 17 \beta$-diol disulfate; E0893, 1,3,5(10)estratrien-3,17 $\alpha$-diol 3 -sulfate (17 $\alpha$-estradiol 3-sulfate); E1050, $1,3,5$ (10)-estratrien-3,17 $\beta$-diol disulfate (17 $\beta$-estradiol disulfate); E4105, 4-estren-17 $\beta$-ol-3-one sulfate (nandrolone sulfate); P3817 $5 \alpha$-pregnan-3 $\alpha$-ol-20-one sulfate (allopregnanolone sulfate); P3865, $5 \alpha$ pregnan-3 $\beta$-ol-20-one sulfate (epiallopregnanolone sulfate); P8200, $5 \beta$-pregnan-3 $\beta$-ol-20-one sulfate (epipregnanolone sulfate); Q1570 4-pregnen-11 $\beta, 21$-diol-3,20-dione 21-sulfate (corticosterone 21-sulfate); Q3383, 4-pregnen-17-ol-3,20-dione sulfate; Q3910, 4-pregnen-11 $\beta$, 17,21-triol3,20-dione 21-sulfate (hydrocortisone 21 -sulfate). Stock solutions ( $100 \mathrm{~mm}$ ) of the sulfated steroids were made using either water or methanol and stored at $4^{\circ} \mathrm{C}$. Before each experiment, the stock solutions were further diluted with carboxygenated $\left(95 \% \mathrm{O}_{2}, 5 \% \mathrm{CO}_{2}\right)$ Ringer's solution for a final concentration of $10 \mu \mathrm{M}$. Methanol concentration never exceeded $0.01 \%$ in the final solutions. 2-Heptanone (Sigma-Aldrich) was made by serial dilution into Ringer's, and tested at both $10^{-7} \mathrm{M}$ and $10^{-5} \mathrm{M}$ with identical results. MHC peptide ligands AAPDNRETF and SYFPEITHI (PolyPeptide Laboratories) and $\mathrm{fMLF}$ and Lipoxin (Sigma-Aldrich) were tested at $1 \mu \mathrm{M}$.

Physiology. At the start of the experiment, the mouse was killed under $\mathrm{CO}_{2}$ and decapitated, and the VNOs were removed quickly and placed in cold Ringer's solution. The VNO epithelium was carefully removed from the bony capsule, and the tubular corpus cavernosum was separated from the epithelium. The tissue was adhered to a nitrocellulose membrane (Millipore) such that the dendrite layer was on the top. The nitrocellulose membrane was placed on a custom chamber and placed under the OCPI microscope. The tissue was continuously perfused with Ringer's solution, alternating with a given stimulus, all at $35^{\circ} \mathrm{C}$. The stimulus and flush were delivered through a custom computer controlled ValveLink fluid handling system (Automate Scientific).

Each stimulus was applied for $30 \mathrm{~s}$ following by a $60 \mathrm{~s}$ flush (Ringer's solution) period. Forty-frame image $z$-stacks of the neuronal tissue were obtained in $2 \mathrm{~s}$, with a $3 \mathrm{~s}$ pause to save the data. Each battery of stimuli was applied 4 times, with the stimulus order being randomized.
Image registration. Small but significant shifting and warping of the tissue occurred as the experiment progressed. Such shape changes were corrected using custom three-dimensional nonrigid registration software that minimized the mean square error compared with a fixed reference stack.

ROI identification. ROIs were selected manually, each ROI corresponding to a single neuron. No ROIs were drawn in the dendritic knob layer. ROIs were defined unambiguously through their change in fluorescence due to neuronal activity. For Figures 3-5, both spontaneous and stimulus-driven activity were used to identify ROIs. In the two additional preparations shown in Figure 6, we restricted our analysis to activity coincident with stimulus presentation, identifying 992 and 1004 neurons; of these, more than half (527 and 530, respectively) satisfied the criteria for responsiveness and reproducibility.

Functional analysis. For each ROI, the mean intensity was calculated in each stack. A trial was defined as the 4 prestimulus stacks and 5 peristimulus and poststimulus stacks, and the intensity was normalized to the mean prestimulus intensity. Because of a small nonspecific fluorescence increase on each trial (visible in the raw movies, Movies 1 and 2), the average response to Ringer's solution was subtracted from each trace. To calculate the scalar $\triangle F / F$ response for a given trial, we computed the time-averaged $\Delta F / F$. To emphasize the periods where the response was strongest, we used a weighted average, where the weighting was determined with an automated technique: Given the matrix $\mathbf{D}$, where $D_{i j}$ is the $\Delta F / F$ of the $i$ th $R O I$ at the $j$ th time point, we approximated $\mathrm{D}$ as a product of a "spatial" component $\mathbf{u}$, corresponding to the individual ROIs, and a "temporal" component $\mathbf{v}$, using the first term of D's singular value decomposition. The sign of $\mathbf{v}$ was fixed by ensuring it had positive mean (we only observed fluorescence increases), and then the temporal weight vector was calculated by taking the non-negative entries of $\mathbf{v}$ and normalizing them so they summed to 1 . This procedure yielded results which were qualitatively similar to simply averaging over time, but with larger signal-to-noise ratio because it automatically emphasized the time periods of largest $\Delta F / F$. The scalar $\Delta F / F$ values over four trials were used for the $t$ test for reproducibility; in all other cases, the displayed response was the average across trials.

Clustering analysis. Clustering was performed using a variant of mean shift clustering. To ensure consistent categorization, cells from all three experiments shown in Figure 6 were combined, clustered, and then split back into their individual experiments. Clustering individual experiments yielded very similar results to those shown in Figure 6.

Spatial analysis. The spatial coordinates of the ROIs were corrected for the tilted imaging; the new spatial coordinates had perpendicular distance from the surface represented as depth ( $z$ position) (Holekamp et al., 2008; see below).

Surface analysis. Using a single stack, the VNO surface was identified using an automated algorithm: in each "column" of pixels, the location of the surface was identified as a sudden increase in intensity, and across columns the locations were median-filtered over a region $21 \mu \mathrm{m}$ long. Visual inspection was used to verify that this algorithm correctly identified the surface of the tissue in all cases.

\section{Results}

\section{Imaging strategy}

OCPI microscopy works by illuminating the objective's focal plane with a thin sheet of light cast perpendicular to the objective's axis (Fig. $1 A$ ); the objective and illumination optics may be moved together to rapidly scan in three dimensions. A tilted configuration allows surface regions of extended tissues to be visualized. Neuronal activity was visualized via the expression of the calcium-sensitive protein GCaMP2 (Tallini et al., 2006; He et al., 2008). Using OCPI microscopy, we scanned a tissue volume $700 \times 175 \times 200 \mu \mathrm{m}^{3}$ (Fig. $1 \mathrm{~B}$ ) with $5 \mu \mathrm{m} z$-spacing in $2 \mathrm{~s}$; this volume contains several thousand sensory neurons, and includes the entire depth of the vomeronasal epithelium. Typical experiments involved the acquisition of nearly one thousand stacks over a period of $\sim 1 \mathrm{~h}$ (Fig. $1 C$ ). Because excitation is limited to 


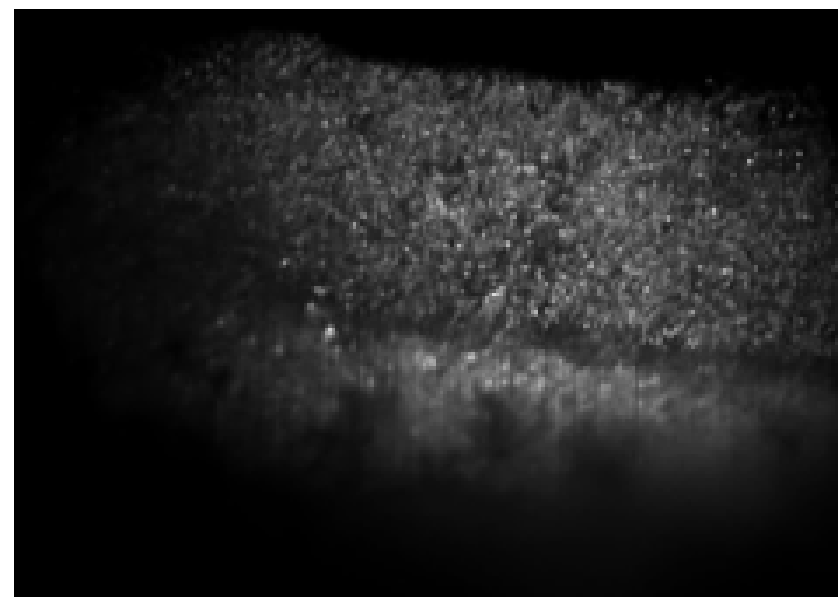

Movie 2. Three-dimensional rendering of fluorescence in whole stacks over time. The same dataset and stimulus trials shown in Movie 2, but with all 40 optical sections rendered as a volume. The "wobble" was introduced computationally as a change in perspective to aid perception of the three-dimensionality of the tissue. "Flashes" corresponding to chemical stimulation can be seen in both the dendritic knobs at the tissue surface, as well as in the cell bodies deeper in the tissue. Movie rendered at 10 stacks per second. Some artifacts of MPEG compression can be seen.

the focal plane, the degree of phototoxicity is manyfold smaller than experienced with confocal microscopy (Holekamp et al., 2008), thus enabling long recording sessions.

\section{Characteristics of responses to VSN ligands}

We stimulated the epithelium with several classes of previously reported VSN ligands. Sulfated steroids, a class of compounds first isolated from mouse urine and which conveys information about the current physiological state of conspecifics (Nodari et al., 2008), have been previously studied by electrical recording (Nodari et al., 2008; Meeks et al., 2010; Arnson and Holy, 2011), but no imaging study has examined responses to these ligands. A set of 12 synthetic sulfated steroids from 4 families-androgens, estrogens, pregnanolones, and glucocorticoids (see Materials and Methods) were presented individually at a concentration of $10 \mu \mathrm{M}$ over repeated randomly interleaved trials with periods of flush between stimuli.

VSNs displayed both spontaneous and robust stimulus-driven activity (Movie 1) resulting in raw changes in fluorescence $(\Delta F / F)$ of magnitudes ranging from a few percent to $>35 \%$ (Fig. $1 C$ ). Activity in hundreds of neurons could be visualized in a single image plane, but the true scale of these recordings can only be appreciated in three-dimensional renderings of the entire volume over time (Movie 2, see also static image in Fig. $1 D$ ). Stimulus responses could be distinguished from spontaneous activity by their clear reproducibility across repeated, interleaved trials (Fig. $1 \mathrm{E}$; see also Fig. 1C).

We also tested several other classes of previously reported VSN ligands, including the volatile 2-heptanone, small peptides that bind to the grooves of $\mathrm{MHC}$ receptors, and formylated peptides. These ligands have been reported to activate a small percentage of neurons in the VNO in several studies (Leinders-Zufall et al., 2000, 2004; Rivière et al., 2009), but other studies have been unable to observe responses to these ligands (Luo et al., 2003; Nodari et al., 2008; Liberles et al., 2009). Because of the methodological difficulties inherent in studying responses that activate only a few percent of neurons in the $\mathrm{VNO}$, the proper interpretation of these negative data has been unclear. We reasoned OCPI microscopy's comprehensive sampling of the intact vomeronasal epithelium might help refine our understanding of the role of these ligands.
We compared, in single optical sections, responses to 2-heptanone and the sulfated steroids P8200, Q1570 and E1050. While robust responses were seen to the sulfated steroids, 2-heptanone proved to be indistinguishable from Ringer's control (Fig. $2 A, B$ ). Similar conclusions were reached upon inspection of the entire imaged volume (Movie 3). Occasional spontaneous activity (Arnson and Holy, 2011) could be distinguished from sensory responses when comparing the same optical section across multiple trials (Movie 3). We also tested the MHC peptide ligands AAPDNRETF and SYFPEITHI (LeindersZufall et al., 2004) and the formylated peptides fMLF and Lipoxin (Rivière et al., 2009). We were likewise unable to find responses in single optical sections (Fig. 2C,D), nor in the imaged volume (Movies 3 ). These stimuli were tested on multiple imaging volumes (2heptanone 0.1 and $10 \mu \mathrm{M}, 5$ volumes across 3 epithelia; MHC peptide ligands, 4 volumes across 3 epithelia; fMLF and Lipoxin, 5 volumes across 3 epithelia) that, for each stimulus, collectively contained an estimated 10,000-25,000 neurons. Inspection of these datasets did not reveal obvious examples of neurons that reproducibly responded to any of these nonsteroid stimuli.

Given the robust stimulus responses to sulfated steroids, we focused our efforts on characterizing the cell-by-cell patterns of sensory responses to sulfated steroids.

\section{Sulfated steroids activated a large fraction of neurons in the apical VNO}

Color-coded representations of the responses to multiple stimuli (Fig. 2E,F) suggested that these 12 sulfated steroids activated a sizable percentage of VSNs. We estimated the percentage of responsive neurons in two ways. First, we performed an unbiased pixel-based analysis, computing the maximum response in individual pixels across all 12 sulfated steroids (Fig. $2 G$ ). In the apical regions of the epithelium [where members of the V1R family of receptors are expressed (Dulac and Axel, 1995)], responsive pixels consistently occupied $\sim 15-20 \%$ of the total volume (single frame shown in Fig. 2G; across 36 frames, peak percentage of pixels with $\Delta F / F>5 \%$ was $20 \pm 2 \%$ mean $\pm S D)$. However, because of gaps between neurons (e.g., secretory ducts visible as black patches in Fig. $1 G$ and Movie 1, and the volume occupied by axons and dendrites), this measure likely underestimates the percentage of cells activated by these stimuli.

We therefore also performed a cell-based analysis, identifying individual neurons by their activity. Within a single imaging volume, we identified 2446 VSNs as being active, either spontaneously or due to stimulus, at some point during the recording. A large percentage (795 cells, 33\%) responded with $\Delta F / F>3 \%$ and $p<0.05$ ( $t$ test for reproducibility against Ringer's control) to at least one sulfated steroid. In the apical epithelium, the proportion of responsive cells was $\sim 50 \%$ (Fig. $2 \mathrm{H}$ ). Because activity is used to unambiguously identify cells, and sensory stimulation is an important source of this activity, this likely represents an upper bound.

Thus, both pixel-based and cell-based analyses indicated that these 12 sulfated steroids activated a large fraction-between a quarter and half- of apical VSNs.

\section{VSNs responses to steroids are stereotyped}

Given that the apical V1R family contains $\sim 200$ different genomic sequences, a priori one might expect that $25-50 \%$ of apical neurons would correspond to a specific population expressing on the order of 50-100 distinct receptor types. This implies a large functional diversity, whose overall features would 
be difficult to make sense of by traditional techniques. Indeed, displaying the responses using a random ordering of the recorded cells (Fig. 3) does not suggest any obvious pattern, consistent with expectations of high diversity. However, the number of responsive cells observed in single preparations by OCPI microscopy was severalfold larger than the anticipated diversity of the population. Because multiple examples of each cell type might be observed in single preparations, the patterns of stimulus responses should reveal the functional organization of this sensory system. We consequently performed a detailed quantitative analysis of these response patterns.

The number of neurons responding to each stimulus varied widely (Fig. $4 A$ ). In many cases, individual neurons responded to multiple sulfated steroids (Fig. $4 B)$. To quantify the specificity of responses across the population, we captured each cell-ligand interaction with a single number, the average $\Delta F / F$, and displayed the population responses with each cell represented by a column of intensities (Fig. 4B, left). Remarkably, ordering the cells by response similarity revealed that the majority of responses were accounted for by a modest number of clusters or "processing streams" (Fig. 4C).

To test whether these clusters represent arbitrary divisions or instead capture the intrinsic organization of these sensory responses, we reduced these 13dimensional response vectors to two dimensions using linear discriminant analysis. The top-level two-dimensional projection (Fig. 4D) revealed several large, clearly separated groupings of points, and more focused projections (Fig. $4 E$ ) confirmed the independence of the remaining clusters. Conversely, a principal components analysis of each cluster individually did not reveal obvious further natural divisions in the data (Fig. 5 ), suggesting that these seven clusters provide a faithful representation of the intrinsic structure of the data. Across preparations, the number of cells in each cluster varied somewhat, but the general pattern was consistent (Fig. 6).

\section{Steroid-responsive classes are} spatially localized

A further layer of organization was revealed by analyzing the location of responsive neurons according to their functional class. Consistent with the observation that most responsive neurons were apical, virtually all of the clusters also displayed a strong apical bias (Fig. 7). However, one processing stream, which responded to both pregnanolones and an androgen (cluster 7 in Fig. 4C), was systematically basal (Fig. 7B). Consequently, the
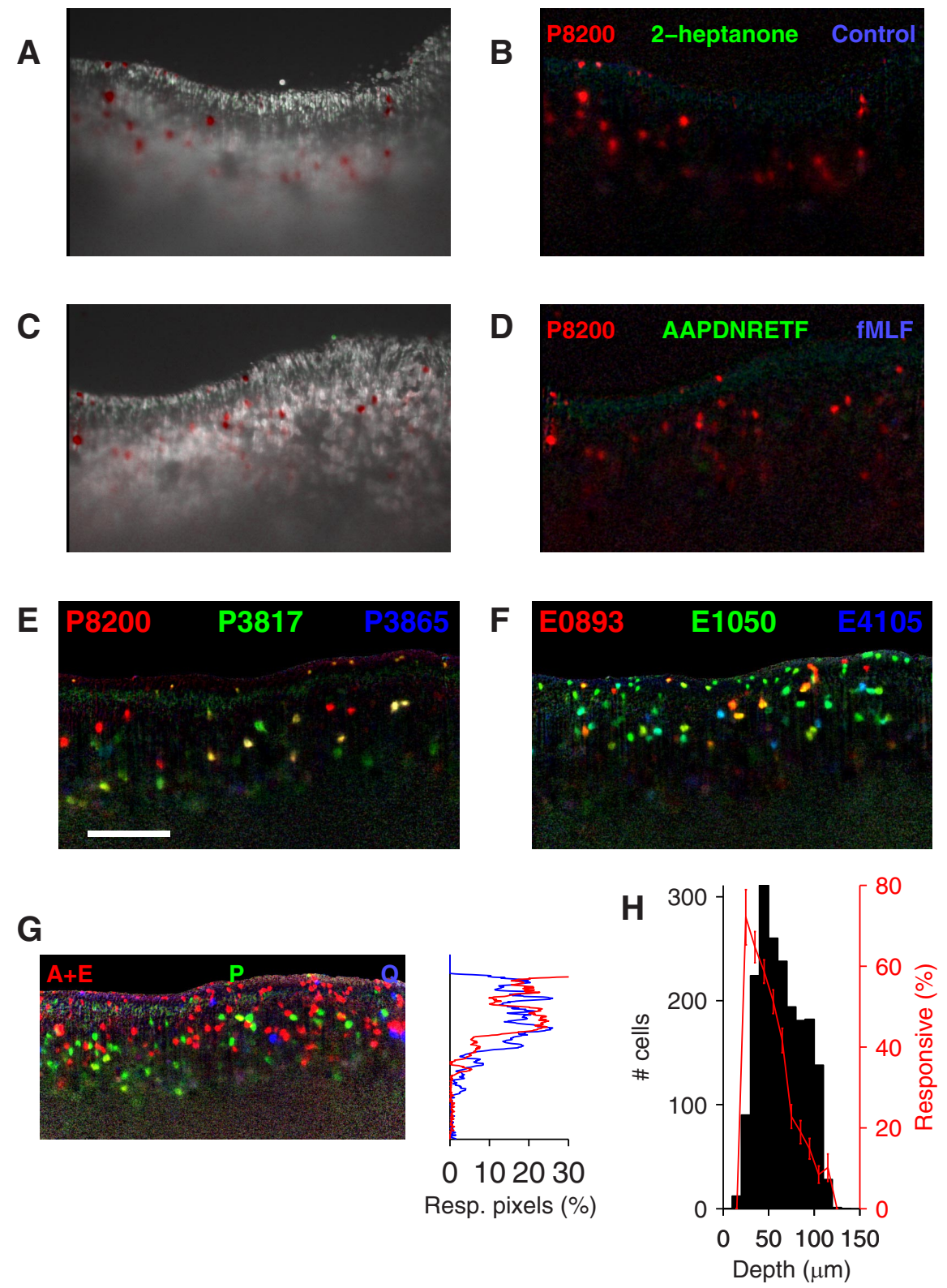

Figure 2. Twelve sulfated steroids collectively activate a large percentage of apical VSNs, but other ligands evoke little or no activity. $\boldsymbol{A}$, Single optical section of VNO, with a red/green color scale used to indicate pixels responsive to the sulfated pregnanolone P8200. $\boldsymbol{B}$, The same optical section, with three overlaid color channels to indicate responses to P8200, 2-heptanone, and Ringer's control. Of these three stimuli, only P8200 caused obvious responses. C, A second preparation, responses to P8200 in a single optical section. D, Same optical section, comparison of responses to $P 8200$ and two putative peptide ligands, AAPDNRETF and fMLF.E, A third preparation, showing a single optical section's responses to three different sulfated pregnanolones. Each pregnanolone causes responses, with some cells responding to more than one of these ligands (indicated by the yellow and white cells). Scale bar, $100 \mu \mathrm{m}$.F, Responses to three sulfated estrogens in this same optical section. $G$, Responses to all 12 steroids as a function of depth within the VNO. Left, a maximum-projection of $\Delta F / F$ across the 12 stimulidisplayed as an RGBimage ( $A$, androgens; $E$, estrogens; $P$, pregnanolones; $Q$, glucocorticoids). Right, the percentage of pixels with $\Delta F / F>5 \%$ for raw image (blue) and one in which the surface was computationally "flattened" by aligning columns of pixels (red). The vertical axis is aligned to the image at the left. $\boldsymbol{H}$, Number of active cells (histogram, left axis) and percentage of these that are steroidresponsive (red line, right axis) as a function of depth below the tissue surface. All panels: responses are average of three trials; color scale from 0 to $15 \% \Delta F / F$ (except $G$, which used $0-10 \% \Delta F / F)$.

functional classifications allowed the identification of types that have a corresponding spatial localization within the epithelium.

\section{Discussion}

Fast calcium imaging

Both electrical and optical techniques allow population recording; for "dense recording" from the majority of neurons in a local 


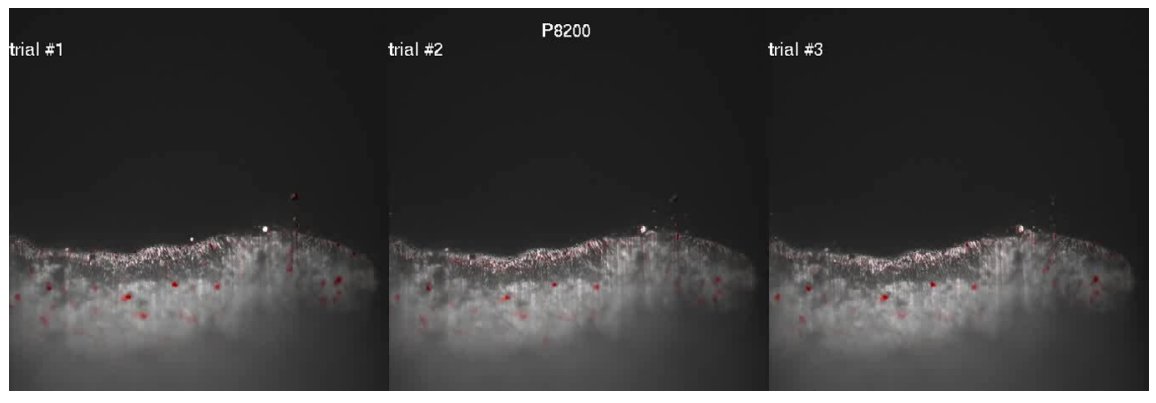

Movie 3. Comparison of responses to sulfated steroids and other ligands. Two separate preparations, one tested with 3 sulfated steroids and the volatile 2-heptanone, the other tested with E1050 and the peptides AAPDNRETF, SYFPEITHI, fMLF, and Lipoxin. The movie plays through all acquired frames in a stack. Average $\Delta F / F$ over the 20 s stimulus presentation is shown (full red is $20 \%$ increase), with separate stimulus trials shown together to permit evaluation of reproducibility. Stimuli were interleaved during delivery. Some spontaneous activity (not reproducible across trials) can be seen in response to any of the stimuli, including the negative control, Ringer's. Some artifacts of MPEG compression can be seen.

volume, optical approaches appear to present a number of advantages. However, one challenge for optical recording is that neurons are distributed throughout a three-dimensional space, requiring methods for fast volumetric imaging. There are several strategies for three-dimensional imaging by point-scanning twophoton microscopy (Wilt et al., 2009), but these approaches are limited by a trade-off among speed, volume, and signal-to-noise ratio that arises from the need to collect a sufficient number of photons from each voxel (Pawley, 2006). To circumvent this obstacle, we recently introduced OCPI microscopy, which records fluorescence emission from a million or more pixels in parallel while providing optical sections with a thickness of a few micrometers (Holekamp et al., 2008). From a single optical section, it is possible to record at speeds of hundreds of frames per second, endowing sensitivity (at least when using the organic indicator Oregon Green BAPTA-1) to single action potentials (Holekamp et al., 2008). However, for our purposes here, a much better use of the speed of OCPI was to collect images from many different optical sections, thereby sampling thousands of neurons in a volume hundreds of micrometers on each side. This approach allowed us to record from neuronal populations that exceed, by a large margin, even the presumptive diversity of the VSN population.

Our results demonstrate that OCPI microscopy enables population recording on an unprecedented scale. Our current imple- mentation of OCPI microscopy allows one to measure activity in several thousand neurons simultaneously. We have successively imaged multiple locations, thereby recording in excess of 10,000 neurons from a single animal in a single day.

The current speed of OCPI microscopy is far from any physical limit (Holekamp et al., 2008), and is set by the rate of data transfer from the camera. Recent and future innovations lead us to expect these numbers to grow considerably in the near future.

\section{Lack of responses to several nonsteroid classes of ligands}

A surprising outcome of our experiments was the lack of responses to several ligands that have been previously reported to activate VSNs (LeindersZufall et al., 2000, 2004; Rivière et al., 2009). It is possible that our recordings, despite their apparently comprehensive nature, somehow missed the activity of these ligands. One scenario is that responses to these ligands were systematically below our threshold for detection, a concern made more likely by the fact GCaMP2, when compared with extracellular recording or organic calcium indicators, has relatively low sensitivity (Mao et al., 2008). This possibility cannot be discounted, although the burst firing of VSNs (Arnson and Holy, 2011) and the abundant spontaneous activity evident in our recordings (Movie 1) make this seem unlikely. Moreover, this same mouse was used in one of the reports (He et al., 2008) supporting the effectiveness of $\mathrm{MHC}$ peptide ligands, indicating that the properties of GCaMP2 cannot account for all of the differences. These results are also consistent with electrophysiology studies (Luo et al., 2003; Nodari et al., 2008) — which are far less comprehensive but have single-spike sensitivity — that also did not find responses to these ligands.

OCPI microscopy permitted such comprehensive recording, from intact vomeronasal epithelium, that the lack of responses cannot plausibly be attributed to undersampling: given 10,000 neurons, the probability of omitting a class representing $0.1 \%$ of the population is $<10^{-4}$. Thus, even quite rare responses, rarer than reported to be responsive to any of these ligands, should have been detected, and so their absence is highly significant. In

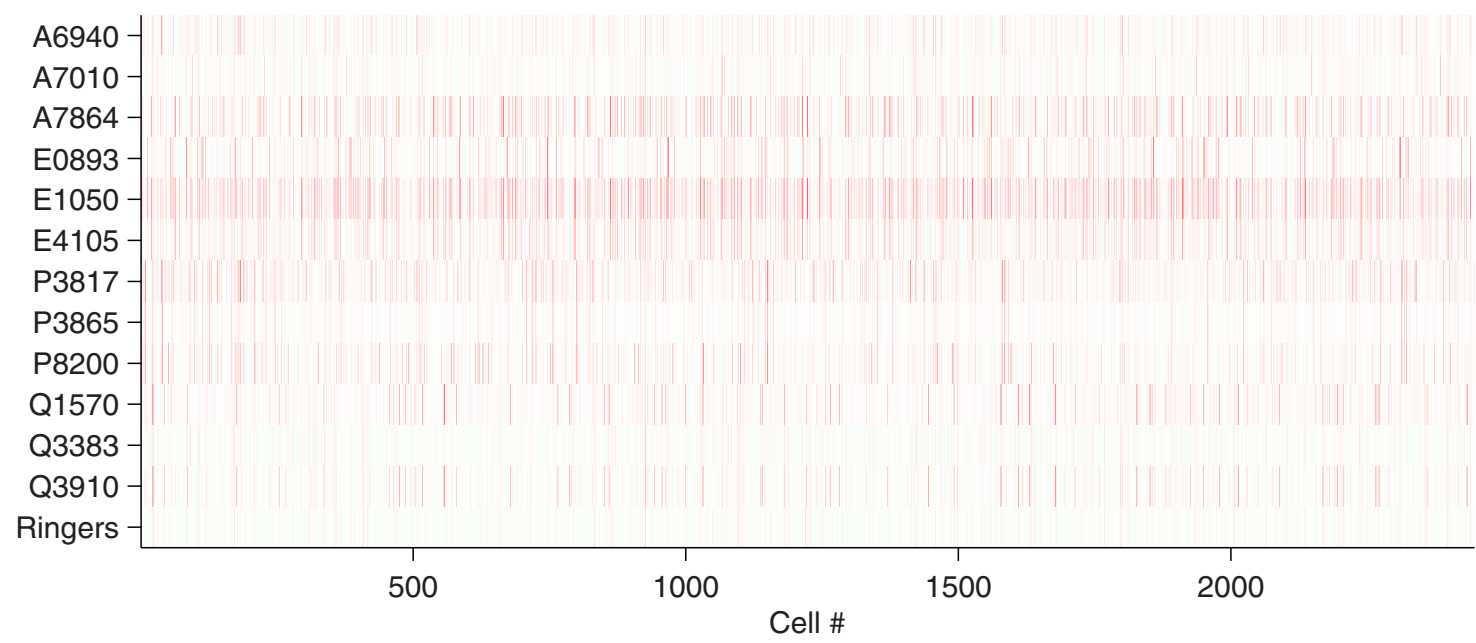

Figure 3. Responses to 12 sulfated steroids and the Ringer's control in 2446 VSNs from a single experiment. Color scale is as shown in Figure $4 B$. 
A

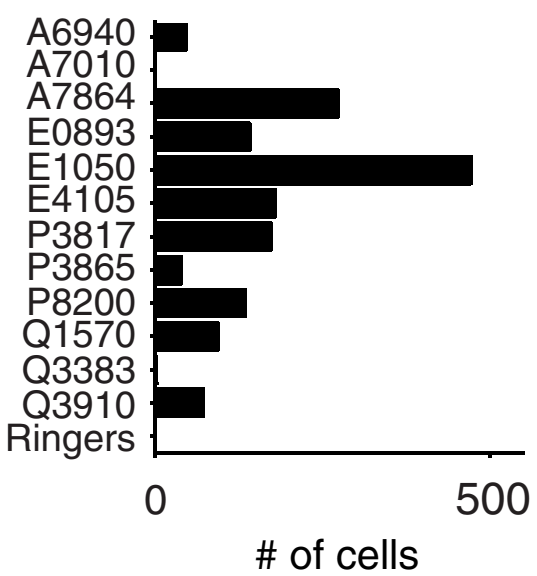

B

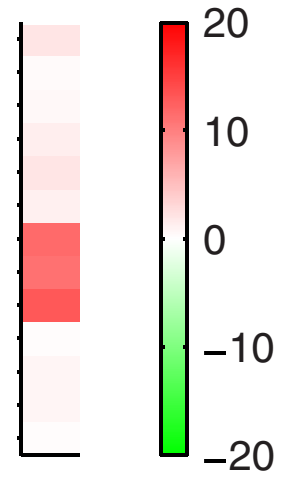

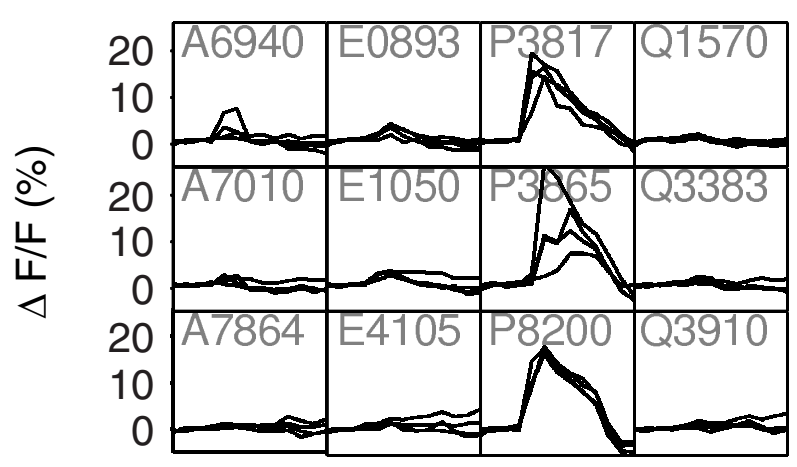

Time

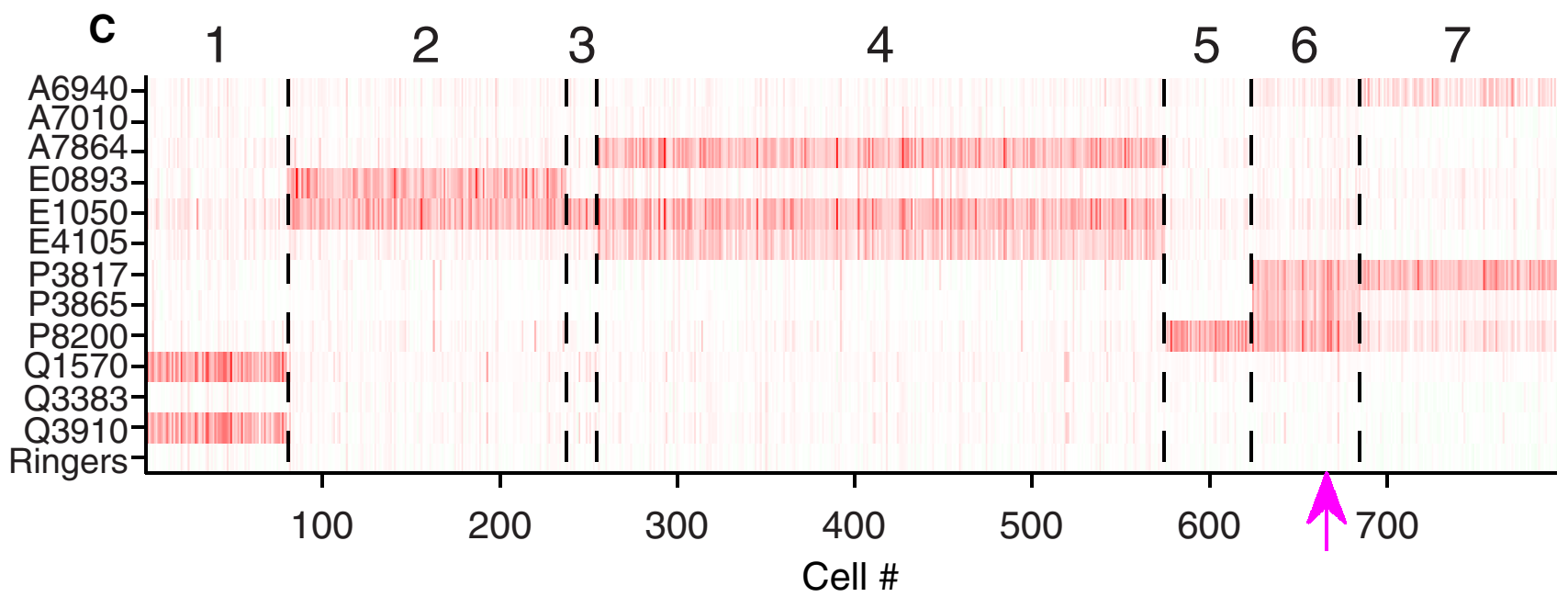

D

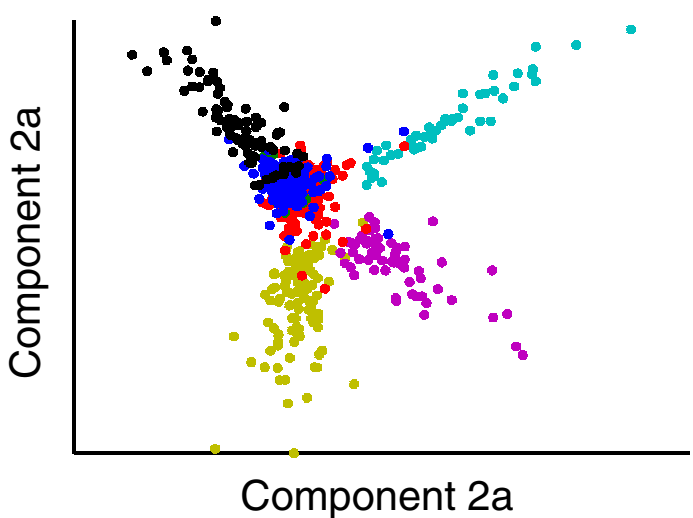

E

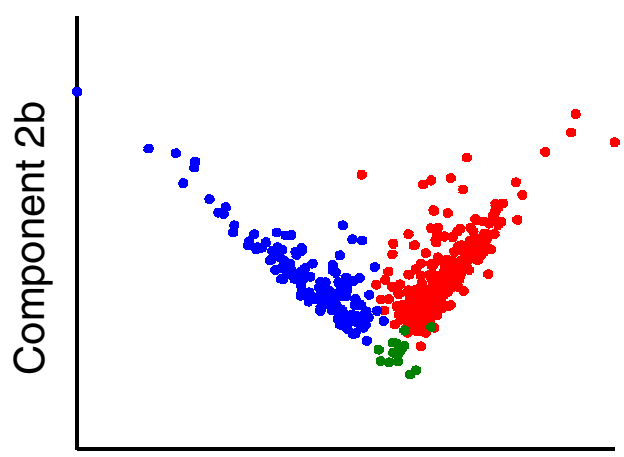

Component 1b

Figure 4. Organization of vomeronasal responses to sulfated steroids. $\boldsymbol{A}$, Histogram of number of cells responding to each stimulus (10 $\mu \mathrm{m}$ ) in a single imaging volume. $\boldsymbol{B}$, A single example cell's responses, plotted as a $\Delta F / F$ color scale (left, stimulus labeling as in $\boldsymbol{A}$ ) and as traces on individual trials (right). $\boldsymbol{C}$, Clustered organization of responses across all 795 responsive cells. Each cell displayed as a single column; magenta arrow indicates the example cell in $\boldsymbol{B}$. D. Two-dimensional projection, via linear discriminant analysis, of response data for all clusters (cluster identity indicated by color in legend); each dot represents a single neuron. $\boldsymbol{E}$, Separate two-dimensional projection of just clusters 2, 3, and 4; these clusters appear to overlap in $\boldsymbol{D}$.

addition to exhaustive sampling, another advantage of OCPI microscopy is its low phototoxicity. This allowed us to test every neuron reported here with 50-80 stimulus trials (Fig. 1C), a number which appears to significantly exceed the number of trials in previous studies that have reported positive results with these ligands. The extra statistical certainty afforded by having more trials is a major benefit in a sensory system whose sponta- neous firing properties mimic many aspects of stimulated responses (Arnson and Holy, 2011).

Another possible explanation of the discrepancy is suggested by the observation that odorant receptors from the main olfactory system are sometimes expressed in the VNO (Lévai et al., 2006). Given reports that both 2-heptanone and the MHC peptide ligands activate the main olfactory system (Spehr et al., 2006), it is possible 
Cluster 1
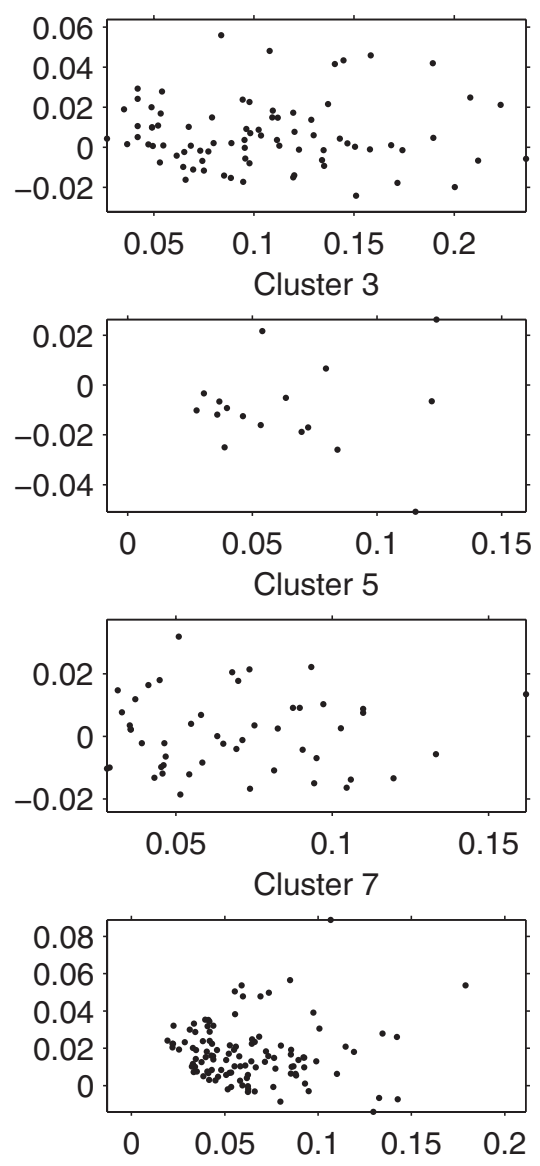

Figure 5. Principal component analysis of the 7 clusters of Figure $4 C$. For each cluster, the first two principal component projections are shown. No obvious natural subdivisions can be seen.

that responses to these ligands were driven by "misexpressed" odorant receptors; if the amount or type of misexpression varies with the mouse strain being used, it could be a source of significant differences among laboratories.

At a minimum, the abundance and robustness of responses to sulfated steroids, particularly when compared with several previously reported classes of ligands, suggests that steroid metabolites may play a particularly central role in vomeronasal sensation.

\footnotetext{
Abundant and stereotyped VSN responses to sulfated steroids The mouse genome contains $\sim 200$ presumed-functional V1R family members (Shi and Zhang, 2007; Young et al., 2010). A collection of just 12 sulfated steroids, delivered at $10 \mu \mathrm{M}$, activated between a quarter and half of all apical cells. The sheer number of these responses demonstrates the centrality of sulfated steroids to vomeronasal sensory coding.

From the size of the V1R family, one might naively expect that this fraction of cells would express between 50 and 100 different receptor types. Previously, we showed by multielectrode array recording (Meeks et al., 2010) that VSN responses can be grouped into processing streams, but the number of streams that could be distinguished was largely limited by the number of neurons sampled. Volumetric calcium imaging did identify new classes of response (e.g., 2 of the 3 pregnanolone-responsive types here were not detected in those previous recordings), but the most
}

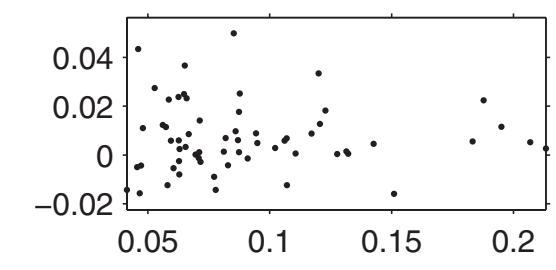

Cluster 2

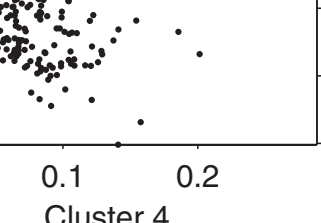

Cluster 4

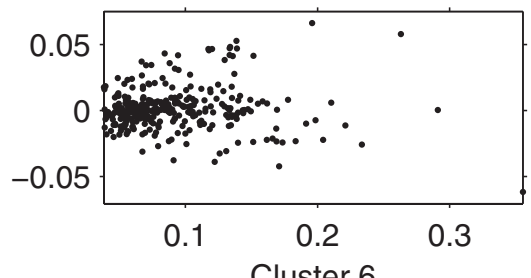

striking and unexpected outcome from calcium imaging is the extreme stereotypy of VSN responses to these 12 sulfated steroids. Even though these represent a small fraction of all possible sulfated steroid structures, in principle 12 compounds should be sufficient to distinguish hundreds or thousands of types even using all-or-none classification of responses $\left(2^{12}=4096\right)$. That so many of the potential response types were not observed suggests that individual receptors have a limited receptive range; that the few observed types accounted for so many cells indicates their disproportionate abundance in the neuroepithelium.

It is worth noting that the processing streams do not typically correspond to cells responding to a single ligand; instead, they are defined only by the pattern of responses across ligands. For example, clusters 2, 3, and 4 contained cells that all responded to E1050; these clusters are distinguished by their responses to the other two E-family compounds. Examples of cells in these clusters can be seen in Figure 2, $E$ and $F$; the distinct processing streams can be recognized visually from the fact that particular color combinations appear repeatedly while others do not appear at all.

What might account for the unexpected degree of order revealed by these large-scale population recordings? One possibility is that each processing stream represents a collection of multiple receptor types with overlapping or redundant functional properties. This possibility is consistent with the observation that, among the families of mammalian and insect odorant receptors, vomeronasal receptors are unusual in possessing a number of distinct subfamilies tightly clustered by amino acid sequence (Rodriguez et al., 2002). Alternatively, it seems possible that these processing streams correspond to single receptor types, but these types are expressed by a large percentage of neurons. Indeed, in certain regions of the mouse main olfactory epithelium, just one receptor type, MOR 28 , is expressed by $\sim 10 \%$ of all olfactory sensory neurons (Tsuboi et al., 1999), and among the V1R receptors, approximately half of the total expression is attributable to the V1Rc and V1Rd subfamilies (Roppolo et al., 2007). This possibility appears to be consistent with recent results identifying individual vomeronasal receptors expressed by cells showing immediate-early gene expression in mice exposed to sulfated steroids (Isogai et al., 2011). While making a precise correspondence is not possible due to differences in stimuli and methodology, our cluster 1 may correspond to V1re2 and V1re6, cluster 2 with V1rf3, and cluster 4 with V1rj2. Because the receptor identification may not have been exhaustive, it is possible that other receptor types with similar response profiles also contribute to these clusters.

The strong apical bias (Fig. 7) suggests that the vast majority of steroid-responsive neurons express members of the V1R family. However, one particular processing stream was found almost exclusively in the basal VNO (Fig. 7). These neurons may express mem- 

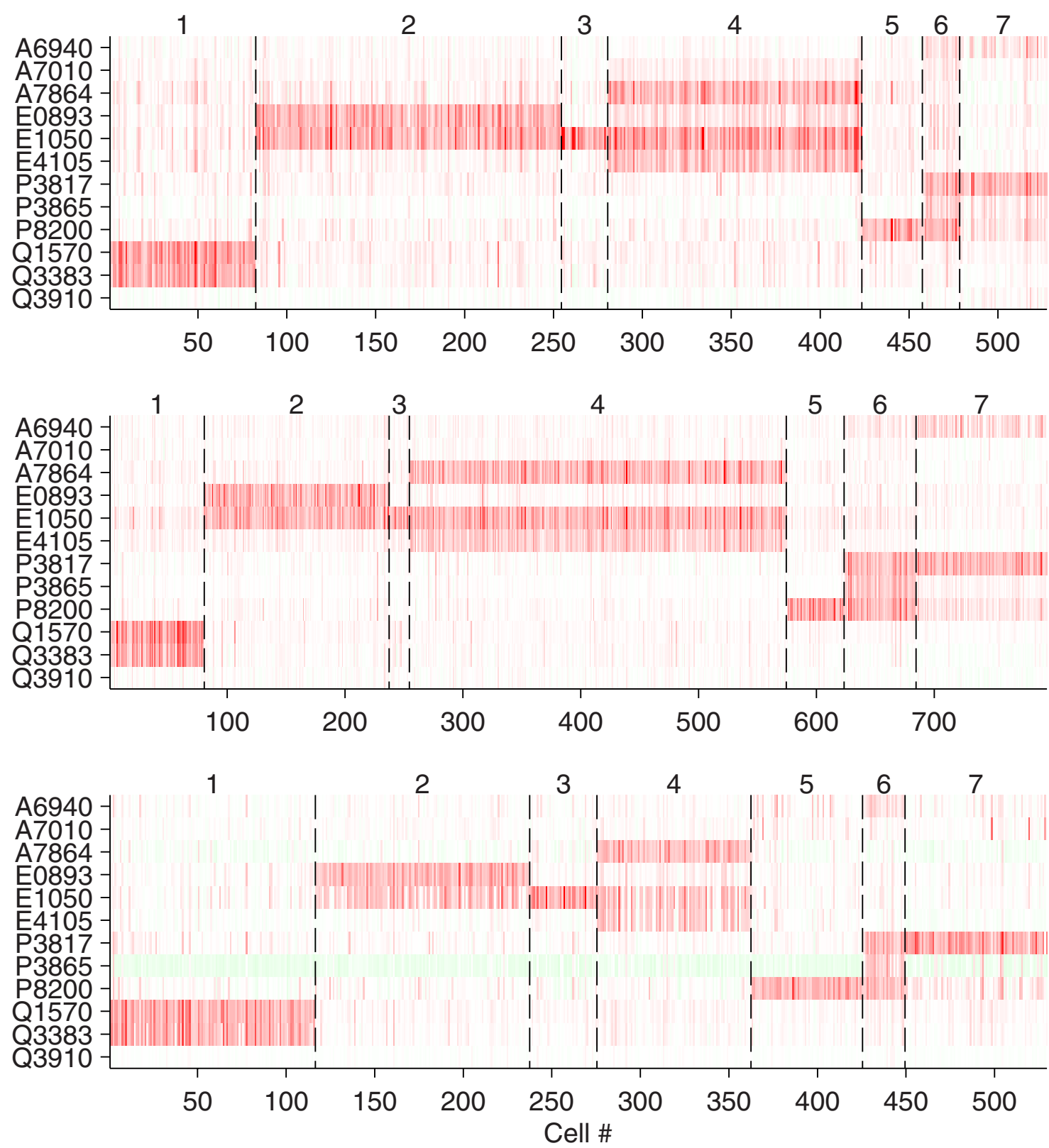

Figure 6. Clustering of reproducibly responsive cells in three different experiments. Color scale is as shown in Figure 4B. By comparison to Figure $4 C$ (reproduced as the middle panel here), note the omission of Q3383, which was not tested in the third experiment (and was not active in the other two experiments).

bers of the V2R family of receptors, indicating that sulfated steroids are detected by members of both major families of vomeronasal receptors. Alternatively, because the location of the V1R/V2R boundary is slightly variable (Herrada and Dulac, 1997; Ishii and Mombaerts, 2008), these neurons may represent a population of deep V1R-expressing neurons.

\section{Conclusions}

OCPI microscopy's comprehensive sampling revealed unexpected stereotypy and spatial organization in the sensory representations of the vomeronasal organ. In neuronal circuits, functional diversity is the rule rather than the exception. It seems likely

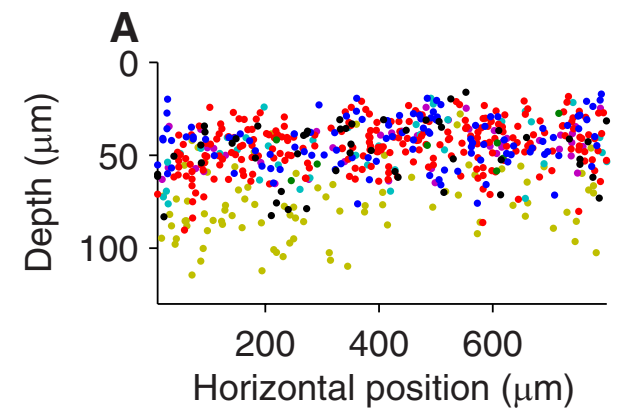

B

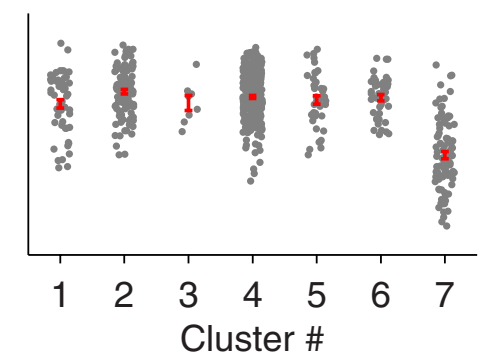

Figure 7. One processing stream is expressed basally within the vomeronasal epithelium. $\boldsymbol{A}$, Scatter plot of cell centers as a function of depth below the tissue surface and the longest imaging axis. Colors indicate cluster number as in Figure $4 D$. Note axes are not of equal scale. $\boldsymbol{B}$, Mean and SEM of depth as a function of cluster number. Cell centroids are plotted in gray, with small random horizontal offsets to reduce overlap. 
that the ability to record on a large scale will yield further new insights about the organization of the nervous system.

\section{References}

Arnson HA, Holy TE (2011) Chemosensory burst coding by mouse vomeronasal sensory neurons. J Neurophysiol 106:409-420.

Buck L, Axel R (1991) A novel multigene family may encode odorant receptors: a molecular basis for odor recognition. Cell 65:175-187.

Dulac C, Axel R (1995) A novel family of genes encoding putative pheromone receptors in mammals. Cell 83:195-206.

He J, Ma L, Kim S, Nakai J, Yu CR (2008) Encoding gender and individual information in the mouse vomeronasal organ. Science 320:535-538.

Herrada G, Dulac C (1997) A novel family of putative pheromone receptors in mammals with a topographically organized and sexually dimorphic distribution. Cell 90:763-773.

Holekamp TF, Turaga D, Holy TE (2008) Fast three-dimensional fluorescence imaging of activity in neural populations by objective-coupled planar illumination microscopy. Neuron 57:661-672.

Hsu FF, Nodari F, Kao LF, Fu X, Holekamp TF, Turk J, Holy TE (2008) Structural characterization of sulfated steroids that activate mouse pheromone-sensing neurons. Biochemistry 47:14009-14019.

Ishii T, Mombaerts P (2008) Expression of nonclassical class I major histocompatibility genes defines a tripartite organization of the mouse vomeronasal system. J Neurosci 28:2332-2341.

Isogai Y, Si S, Pont-Lezica L, Tan T, Kapoor V, Murthy VN, Dulac C (2011) Molecular organization of vomeronasal chemoreception. Nature 478:241-245.

Leinders-Zufall T, Lane AP, Puche AC, Ma W, Novotny MV, Shipley MT, Zufall F (2000) Ultrasensitive pheromone detection by mammalian vomeronasal neurons. Nature 405:792-796.

Leinders-Zufall T, Brennan P, Widmayer P, S PC, Maul-Pavicic A, Jäger M, Li XH, Breer H, Zufall F, Boehm T (2004) MHC class I peptides as chemosensory signals in the vomeronasal organ. Science 306:1033-1037.

Lévai O, Feistel T, Breer H, Strotmann J (2006) Cells in the vomeronasal organ express odorant receptors but project to the accessory olfactory bulb. J Comp Neurol 498:476-490.

Liberles SD, Horowitz LF, Kuang D, Contos JJ, Wilson KL, Siltberg-Liberles J, Liberles DA, Buck LB (2009) Formyl peptide receptors are candidate chemosensory receptors in the vomeronasal organ. Proc Natl Acad Sci U S A 106:9842-9847.

Luo M, Fee MS, Katz LC (2003) Encoding pheromonal signals in the accessory olfactory bulb of behaving mice. Science 299:1196-1201.

Mao T, O'Connor DH, Scheuss V, Nakai J, Svoboda K (2008) Characterization and subcellular targeting of GCaMP-type genetically-encoded calcium indicators. PLoS One 3:e1796.
Meeks JP, Arnson HA, Holy TE (2010) Representation and transformation of sensory information in the mouse accessory olfactory system. Nat Neurosci 13:723-730.

Nodari F, Hsu FF, Fu X, Holekamp TF, Kao LF, Turk J, Holy TE (2008) Sulfated steroids as natural ligands of mouse pheromone-sensing neurons. J Neurosci 28:6407-6418.

Pawley JB, editor (2006) Handbook of Biological Confocal Microscopy, Ed 3. New York: Springer.

Rivière S, Challet L, Fluegge D, Spehr M, Rodriguez I (2009) Formyl peptide receptor-like proteins are a novel family of vomeronasal chemosensors. Nature 459:574-577.

Rodriguez I, Del Punta K, Rothman A, Ishii T, Mombaerts P (2002) Multiple new and isolated families within the mouse superfamily of V1r vomeronasal receptors. Nat Neurosci 5:134-140.

Roppolo D, Vollery S, Kan CD, Lüscher C, Broillet MC, Rodriguez I (2007) Gene cluster lock after pheromone receptor gene choice. EMBO J 26:3423-3430.

Shi P, Zhang J (2007) Comparative genomic analysis identifies an evolutionary shift of vomeronasal receptor gene repertoires in the vertebrate transition from water to land. Genome Res 17:166-174.

Spehr M, Kelliher KR, Li XH, Boehm T, Leinders-Zufall T, Zufall F (2006) Essential role of the main olfactory system in social recognition of major histocompatibility complex peptide ligands. J Neurosci 26:1961-1970.

Tallini YN, Ohkura M, Choi BR, Ji G, Imoto K, Doran R, Lee J, Plan P, Wilson J, Xin HB, Sanbe A, Gulick J, Mathai J, Robbins J, Salama G, Nakai J, Kotlikoff MI (2006) Imaging cellular signals in the heart in vivo: Cardiac expression of the high-signal $\mathrm{Ca}^{2+}$ indicator GCaMP2. Proc Natl Acad Sci U S A 103:4753-4758.

Touhara K, Vosshall LB (2009) Sensing odorants and pheromones with chemosensory receptors. Annu Rev Physiol 71:307-332.

Tsuboi A, Yoshihara S, Yamazaki N, Kasai H, Asai-Tsuboi H, Komatsu M, Serizawa S, Ishii T, Matsuda Y, Nagawa F, Sakano H (1999) Olfactory neurons expressing closely linked and homologous odorant receptor genes tend to project their axons to neighboring glomeruli on the olfactory bulb. J Neurosci 19:8409-8418.

Wilt BA, Burns LD, Wei Ho ET, Ghosh KK, Mukamel EA, Schnitzer M] (2009) Advances in light microscopy for neuroscience. Annu Rev Neurosci 32:435-506.

Young JM, Massa HF, Hsu L, Trask BJ (2010) Extreme variability among mammalian V1R gene families. Genome Res 20:10-18.

Yu CR, Power J, Barnea G, O’Donnell S, Brown HE, Osborne J, Axel R, Gogos JA (2004) Spontaneous neural activity is required for the establishment and maintenance of the olfactory sensory map. Neuron 42:553-566. 